\title{
Bandwidth-Efficient Pilot-Symbol-Aided Technique for Multipath-Fading Channels
}

\author{
Man-Hung Ng and Sing-Wai Cheung
}

\begin{abstract}
Pilot-symbol-aided (PSA) transmission is one of the effective methods to combat multipath fading in digital mobile communications systems. In the PSA systems, redundant bandwidth and power are required to transmit the pilot symbols. In this paper, we propose a novel fading estimation technique that requires a very low bandwidth redundancy in the PSA systems. The proposed technique uses simple linear interpolation on the pilot symbols and the detected data symbols to obtain the estimates of the channel fading effects. Monte Carlo computer simulation has been carried out to study the effects of the technique on the bit error rate performance of 16-ary quadrature amplitude modulation in the flat and the frequency-selective Rayleigh-fading channels corrupted with cochannel interference and additive white Gaussian noise. Results have shown that the proposed technique can, at the expense of a little power efficiency, significantly improve the bandwidth efficiency of the PSA systems using receivers with low complexity and latency.
\end{abstract}

Index Terms-Bandwidth efficiency, fading estimation, pilot-symbol-aided (PSA) transmission, Rayleigh fading, 16 QAM.

\section{INTRODUCTION}

$\mathbf{M}$ ULTIPATH-fading distortion is a major problem in digital mobile communications systems because it severely degrades the error rate performances and very often causes error floors to the systems. Pilot-symbol-aided (PSA) transmission has been found to be an effective method to combat multipath fading for digital modulation in the mobile environments [1]-[8]. In a PSA system, pilot symbols from a known pseudorandom-symbol sequence are multiplexed with data symbols for transmission. The receiver has a prior knowledge of the pilot symbol sequence and so can extract the pilot symbols from the received signal and subsequently estimate and compensate for the fading effects on the data symbols. The fading estimates on the data symbols are obtained by interpolating the fading estimates on the pilot symbols. Conventional PSA fading estimation techniques make use of only the pilot symbols but ignore the data symbols [1]-[4]. These estimation techniques require the use of high-order interpolating functions, where numerous pilot symbols have to be buffered and then interpolated to give the fading estimates on the data symbols. Otherwise, when the fading rate is fast compared to the pilot symbol rate, the fading estimates become less accurate and high error floors are incurred to the systems [2], [4], [8], [9]. However, the use of high-order interpolating functions greatly increases the complexity of the receivers.

Manuscript received July 11, 1998; revised July 25, 2000.

The authors are with the Department of Electrical and Electronic Engineering, The University of Hong Kong, Hong Kong (e-mail: mhng@eee.hku.hk; swcheung@eee.hku.hk).

Publisher Item Identifier S 0018-9545(01)07004-9.
More importantly, especially when the pilot symbol rate is slow, the latency due to pilot symbol buffering is greatly lengthened using high-order interpolating functions, and this is undesirable for voice services in mobile communications. If the fading effects are to be estimated more accurately using low-order interpolating functions, the pilot symbols need to be sent more frequently, and this increases the bandwidth redundancy of the system since more bandwidth is used to transmit the pilot symbols [4], [10]. Different estimation techniques that make use of the data symbols as well as the pilot symbols have also been proposed [11]-[13]. Compared to the conventional techniques, these data-aided techniques provide better performances in fast fading environments. However, these techniques require the use of high-order smoothing filters to reduce the noise effects on the fading estimates obtained using the data symbols, and thus add computational load and delay to the systems.

In a PSA system, redundant bandwidth and power are required to transmit the pilot symbols. Based on the sampling theory, a fading process with a maximum Doppler spread of $f_{D}$ could be sampled without distortion using the Nyquist rate of $2 / f_{D}$ [4], [6]. This means that if $1 / T_{F}$ is the pilot symbol rate, then $f_{D} T_{F}=0.5$ would be sufficient to obtain accurate fading estimates. However, using the second-order Gaussian interpolating function in the Rayleigh-fading channels with the normalized fading rates of $f_{D} T=5 \times 10^{-3}$ and $f_{D} T=2 \times 10^{-2}$, where $T$ is the channel symbol period, researchers have suggested the frame lengths of 16 [4] and 8 [10], respectively, resulting in $f_{D} T_{F}=0.08$ and $f_{D} T_{F}=0.16$, both of which are substantially smaller than $f_{D} T_{F}=0.5$. Note that the bandwidth redundancies of the PSA systems with these frame lengths are $6.25 \%$ and $12.5 \%$, respectively.

In this paper, we propose a novel PSA technique that requires a very low bandwidth redundancy to transmit the pilot symbols. The proposed technique uses simple linear interpolation to obtain the fading estimates from the pilot symbols as well as the detected data symbols. As the data symbols are also used to provide samples of the channel fading process, the pilot symbol rate is no longer constrained by the Nyquist rate and thus the bandwidth redundancy of the PSA system can be greatly reduced. Since linear interpolation is used, the complexity and latency of the receiver can also be greatly reduced. A series of computer simulation has been carried out to assess the effects of the proposed technique on the bit error rate (BER) performance of 16-ary quadrature amplitude modulation (16 QAM) in the flat and the frequency-selective Rayleigh-fading environments, with and without the distortions of cochannel interference (CCI) and additive white Gaussian noise (AWGN). Results have shown that the proposed technique can, at the expense of 


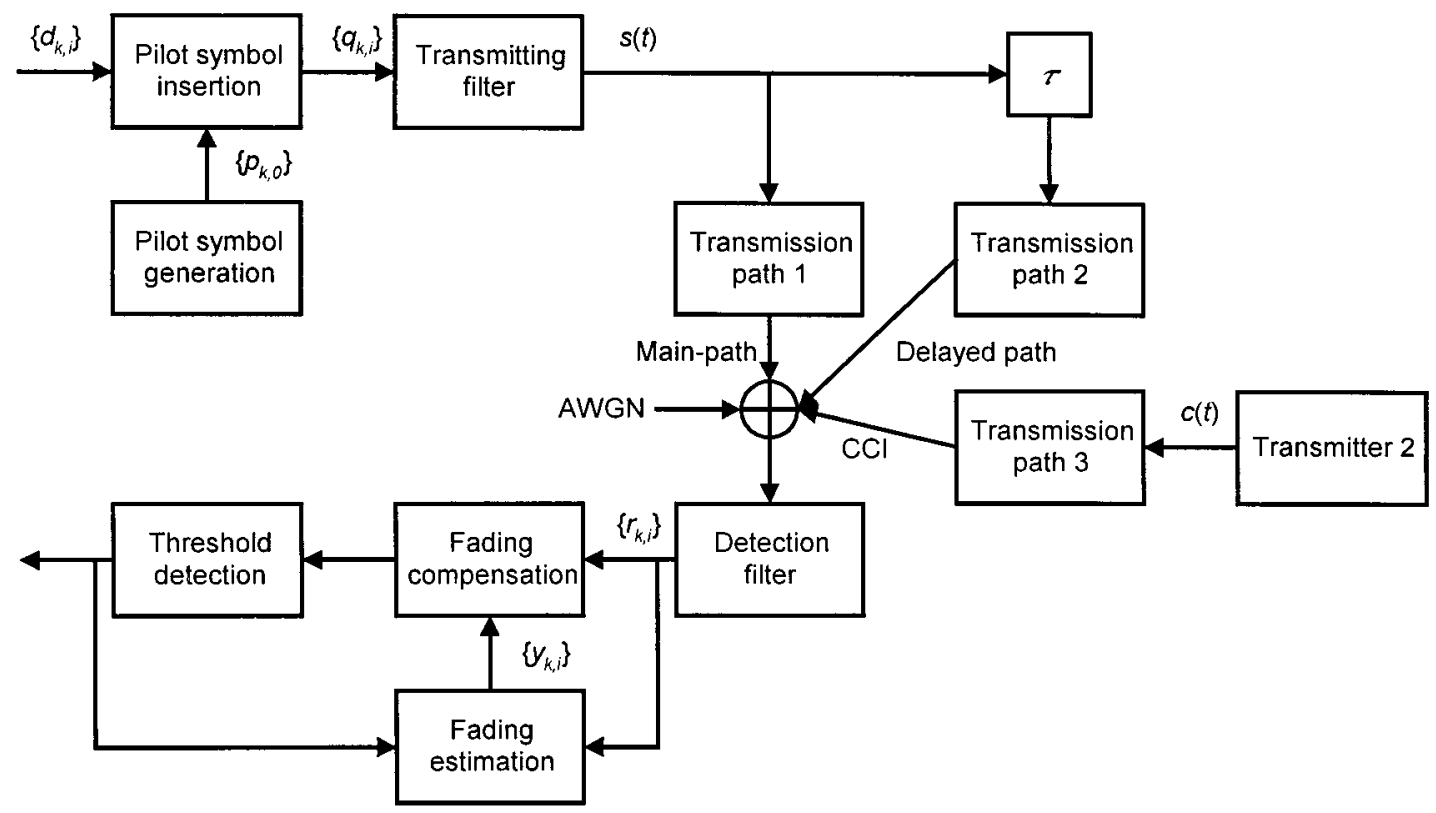

Fig. 1. System block diagram.

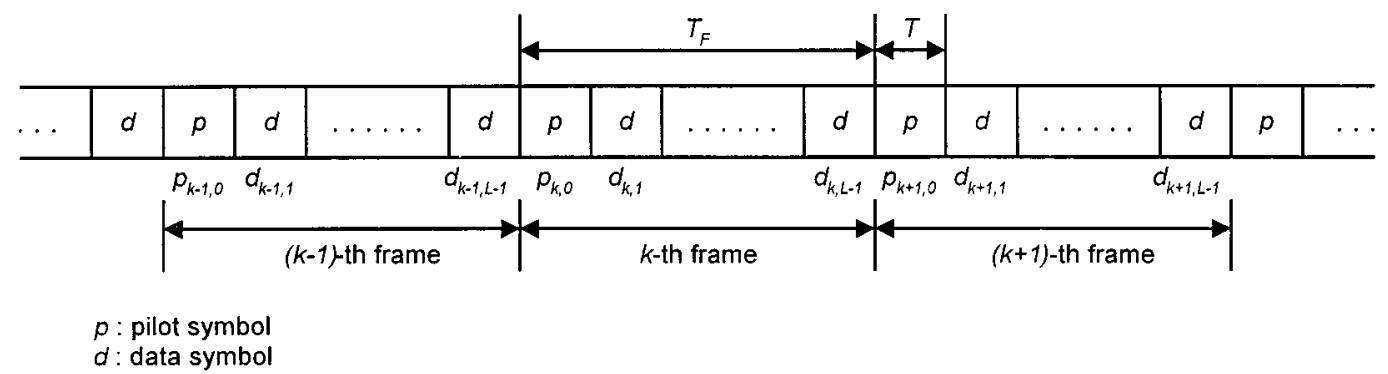

Fig. 2. Frame structure.

a little power efficiency, significantly reduce the bandwidth redundancy required for using low-order interpolating functions to obtain accurate fading estimates. It has also been shown that the proposed technique can significantly lower the error floors of the PSA system caused by using low-order interpolating functions.

The remainder of this paper is organized into four parts. Section II shows how the system and fading process are modeled. Section III describes the proposed technique and the mathematical basis behind it. Simulation results together with discussions are given in Section IV. Finally, Section V presents the conclusions.

\section{SySTEM MODEL}

The baseband equivalent block diagram of the land mobile communications system used in the study is shown in Fig. 1. At the transmitter, the data symbols $\left\{d_{k, i}\right\}$ are possible vectors on the rectangular 16 QAM signal constellation. A pilot symbol from a known pseudorandom-symbol sequence $\left\{p_{k, 0}\right\}$ is multiplexed with every $(L-1)$-data symbols to form an $L$-symbol frame with a frame duration of $T_{F}$, as shown in Fig. 2. A pseudorandom sequence of pilot symbols is used to avoid transmitting tones at the multiples of the pilot symbol frequency $1 / T_{F}[1]$.
To minimize the error in fading estimation due to additive noise in the pilot symbols, these pilot symbols are chosen from those vectors with the highest energy level in the signal constellation [4]. At time $t=n T$, the data or pilot symbol $q_{n}$ is used to form an impulse $q_{n} \delta(t-n T)$, which is fed to the transmitting filter with an impulse response $a(t)$ to produce the signal at the filter output

$$
s(t)=\sum_{n} q_{n} a(t-n T) .
$$

Each of the transmission paths in Fig. 1 introduces Rayleighfading distortion to the corresponding input signal and is modeled as shown in Fig. 3, where the input signal is multiplied by the complex-valued waveform

$$
b(t)=b^{\prime}(t)+j b^{\prime \prime}(t)
$$

to give the corresponding Rayleigh faded signal. The waveforms $b^{\prime}(t)$ and $b^{\prime \prime}(t)$ are two statistically independent zero-mean Gaussian processes, each with the power spectral density of [14]

$$
|B(f)|^{2}= \begin{cases}\frac{\sigma^{2}}{\pi \sqrt{f_{D}^{2}-f^{2}}}, & |f| \leq f_{D} \\ 0, & |f|>f_{D}\end{cases}
$$




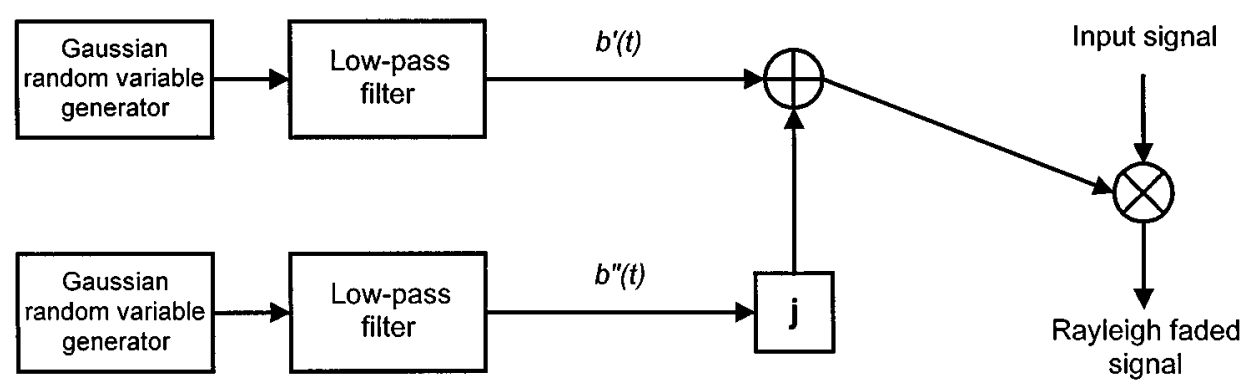

Fig. 3. Rayleigh-fading model.

where

$|B(f)|$
$\sigma^{2}$

$f_{D}$ amplitude of the frequency response $B(f)$; common variance of $b^{\prime}(t)$ and $b^{\prime \prime}(t)$; maximum Doppler spread of the faded signal caused by the mobile motion and given by

$$
f_{D}=v / \lambda
$$

with $v$ the velocity of the mobile and $\lambda$ the wavelength of the signal carrier.

The combination of the main-path signal $s(t)$ and the delayed signal $s(t-\tau)$, where $\tau$ is the delay between the two signals, accounts for the frequency-selective fading of the channel [6]. The cochannel signal $c(t)$ in Fig. 1 is assumed to have the same structure as the main-path signal $s(t)$ and to be both frame-aligned and symbol-aligned with $s(t)$. Stationary AWGN with one-sided power spectral density of $N_{0}$ is added at the input of the receiver. Thus the signal at the receiver input consists of the main-path signal, the delayed signal, CCI, and AWGN. The received signal is filtered by a detection filter, which is taken to have the same impulse response $a(t)$ as the transmitting filter at the transmitter, so that the signal at the filter output is given by

$$
r(t)=[s(t) y(t)+s(t-\tau) x(t)+c(t) v(t)] \otimes a(t)+g(t)
$$

where

$\otimes \quad$ convolution process;

$y(t), x(t)$ and $v(t)$ statistically independent fading distortions introduced into the main-path signal, the delayed signal, and the co-channel signal, respectively;

$g(t) \quad$ filtered noise waveform.

The baseband signal $r(t)$ is sampled in synchronism at the time instants $\{n T\}$. Assume that the fading rate is slow compared to the symbol rate, and so the intersymbol interference (ISI) caused by the fading process can be neglected [2]. Also assume $a(t) \otimes a(t)=1$ at time $t=0$, so the sample of the filter output at time $t=n T$ is given by

$$
r_{n}=q_{n} y_{n}+w_{n}
$$

where $y_{n}$ represents the fading effect introduced into the symbol $q_{n}$ by the main-path and $w_{n}$ represents the combined additive distortion effect caused by the delayed path, CCI, and AWGN. Since the transmitted pilot symbols are known at the receiver, in the absence of the delayed path, CCI, and AWGN, the fading effect $y_{n}$ on the pilot symbol $q_{n}$ is computed as

$$
y_{n}=r_{n} / q_{n} \text {. }
$$

However, in the presence of the delayed path, CCI, and AWGN, only an estimate of $y_{n}$ can be obtained as

$$
\hat{y}_{n}=r_{n} / q_{n}=y_{n}+w_{n} / q_{n}
$$

which is used to estimate the fading effects on the received data symbols. The proposed estimation technique is described in the following section.

\section{FADING ESTIMATION}

\section{A. Basic Analysis}

For simplicity, it is assumed that frame synchronization has been achieved. The frame structure of the transmitted symbol sequence is shown in Fig. 2. The received symbol at the $i$ th position of the $k$ th frame can be written as

$$
r_{k, i}=q_{k, i} y_{k, i}+w_{k, i}
$$

where

$q_{k, i}$ pilot symbol for $i=0$ and a data symbol for $i=$ $1, \ldots, L-1$;

$y_{k, i} \quad$ fading effect in the main path;

$w_{k, i}$ additive distortion effect due to the delayed path, CCI, and AWGN.

The fading effects $y_{k, 0}$ and $y_{k+1,0}$ on the pilot symbols $p_{k, 0}$ and $p_{k+1,0}$ in the $k$ th and $(k+1)$ th frames, respectively, are estimated as

$$
\begin{aligned}
\hat{y}_{k, 0} & =r_{k, 0} / p_{k, 0}=y_{k, 0}+w_{k, 0} / p_{k, 0} \\
\hat{y}_{k+1,0} & =r_{k+1,0} / p_{k+1,0}=y_{k+1,0}+w_{k+1,0} / p_{k+1,0}
\end{aligned}
$$

By using linear interpolation, $\hat{y}_{k, 0}$ and $\hat{y}_{k+1,0}$ can be used to estimate the fading effects on the data symbols in the $k$ th frame. For example, the fading effects on the first and the last data symbols of the $k$ th frame can be estimated, respectively, as

$$
\begin{aligned}
\hat{y}_{k, 1} & =\left[(L-1) \hat{y}_{k, 0}+\hat{y}_{k+1,0}\right] / L \\
\hat{y}_{k, L-1} & =\left[\hat{y}_{k, 0}+(L-1) \hat{y}_{k+1,0}\right] / L .
\end{aligned}
$$

The instantaneous channel signal-to-noise ratio (SNR) of a data symbol within a frame can be written as $\left|y_{k, i}\right|^{2} E_{b} / N_{0}$ [15], where $E_{b}$ is the average transmitted energy per information 
bit. The estimated instantaneous channel SNR can therefore be written as

$$
\gamma_{k, i}=\left|\hat{y}_{k, i}\right|^{2} E_{b} / N_{0}
$$

Both (11a) and (11b) can be used for fading estimation, depending on whether the first data symbol or the last data symbol of the frame is selected. However, we propose to use the equation that can give a higher estimated instantaneous channel SNR. It can be seen that the numerators of both (11a) and (11b) consist of the fading estimates $\hat{y}_{k, 0}$ and $\hat{y}_{k+1,0}$, with one of them multiplied by the same factor of $(L-1)$. Clearly, the equation with the larger fading estimate, $\hat{y}_{k, 0}$ or $\hat{y}_{k+1,0}$, multiplied by the factor of $(L-1)$, should have a larger numerator, so producing a larger estimate of the adjacent fading effect, $\hat{y}_{k, 1}$ or $\hat{y}_{k, L-1}$, and resulting in a larger estimated instantaneous channel SNR of the adjacent data symbol, $\gamma_{k, 1}$ or $\gamma_{k, L-1}$, respectively, as can be seen from (12). Therefore, selecting the equation with the larger fading estimate multiplied by $(L-1)$ results in a higher estimated instantaneous channel SNR of the adjacent data symbol, $\gamma_{k, 1}$ or $\gamma_{k, L-1}$, and so leads to more accurate fading estimation and data detection.

\section{B. Proposed Technique}

From the above analysis, we propose a fading estimation technique that operates on the data symbols from the outside edges of the frame toward the middle, with the order determined by the estimated instantaneous channel SNR of the symbols. The proposed technique starts with the fading estimates of $\hat{y}_{k, 0}$ and $\hat{y}_{k+1,0}$ using (10a) and (10b), respectively, and then computes the magnitudes of $\left|\hat{y}_{k, 0}\right|$ and $\left|\hat{y}_{k+1,0}\right|$. Depending on whether $\left|\hat{y}_{k, 0}\right|$ or $\left|\hat{y}_{k+1,0}\right|$ is larger, (11a) or (11b), respectively, is used for initial estimation of the fading effect $y_{k, 1}$ or $y_{k, L-1}$. That is, if $\left|\hat{y}_{k, 0}\right|$ is larger than $\left|\hat{y}_{k+1,0}\right|$, the value $\hat{y}_{k, 1}$ is selected to be computed using (11a); otherwise, the value $\hat{y}_{k, L-1}$ is selected to be computed using (11b) instead. The result of this selection determines the next data symbol to be operated. As described previously, selecting the larger fading estimate results in a higher estimated instantaneous channel SNR of the adjacent symbol. Thus this process ensures that the data symbol to be operated will have a higher estimated instantaneous channel SNR. Without loss of generality, we assume here that $\left|\hat{y}_{k, 0}\right|$ is larger than $\left|\hat{y}_{k+1,0}\right|$ and so $\hat{y}_{k, 1}$ is computed.

The estimate $\hat{y}_{k, 1}$ is then used to compensate for the fading effect on the received data symbol $r_{k, 1}$ by

$$
\hat{r}_{k, 1}=r_{k, 1} / \hat{y}_{k, 1} \text {. }
$$

The compensated data symbol $\hat{r}_{k, 1}$ is threshold detected to give the detected data symbol $\hat{d}_{k, 1}$ that is a possible vector on the 16 QAM signal constellation. The detected data symbol $\hat{d}_{k, 1}$ is used to reestimate the fading effect $y_{k, 1}$ as

$$
\tilde{y}_{k, 1}=r_{k, 1} / \hat{d}_{k, 1} \text {. }
$$

The process is repeated, but now with $\hat{y}_{k, 0}$ replaced by the estimated fading effect in the adjacent data symbol $\tilde{y}_{k, 1}$, and for computing the estimate of $y_{k, 2}$ or $y_{k, L-1}$, depending on whether $\left|\tilde{y}_{k, 1}\right|$ or $\left|\hat{y}_{k+1,0}\right|$ is larger. That is, if $\left|\tilde{y}_{k, 1}\right|$ is larger than $\left|\hat{y}_{k+1,0}\right|$, then the value $\hat{y}_{k, 2}$ is selected to be computed by using linear interpolation on $\tilde{y}_{k, 1}$ and $\hat{y}_{k+1,0}$; otherwise, the value $\hat{y}_{k, L-1}$ is selected instead. It should be noted that since $y_{k, 1}$ has a stronger time correlation than $y_{k, 0}$ has with both $y_{k, 2}$ and $y_{k, L-1}$, and the interpolating length is reduced from $L$ to $(L-1)$, the fading estimation error due to linear interpolation by using the estimate of $y_{k, 1}$, instead of $y_{k, 0}$, should be smaller, leading to a more accurate estimate of $y_{k, 2}$ or $y_{k, L-1}$. The selection process here again ensures that the data symbol to be operated will have a higher estimated instantaneous channel SNR. This whole process continues until all the data symbols within the frame have been operated.

To summarize, the proposed technique is processing the received symbols from the outside edges of the pilot frame toward the middle, with the order determined by the symbols with the highest estimated instantaneous channel SNR. The fading effects are estimated by using the pilot symbols and the detected data symbols. The whole process is repeated until all the data symbols within the received signal have been detected.

\section{RESUlts AND Discussions}

\section{A. Basic Assumptions}

Monte Carlo computer simulation tests have been carried out to study the BER performance of the PSA-16 QAM system shown in Fig. 1 using the proposed technique. In all tests, the transfer function of the transmitting and the detection filters in cascade has a raised cosine rolloff of 0.5 . The normalized fading rates of $f_{D} T=2 \times 10^{-2}$ and $f_{D} T=5 \times 10^{-3}$ in the flat and the frequency-selective fading environments, with and without CCI and AWGN, have been tested. For the purpose of comparison, the results using second-order Gaussian interpolation on the pilot symbols solely for fading estimation are also presented. Second-order Gaussian interpolation is used here for comparison because it is a well-adopted low-order interpolating function in related works on the PSA systems [4], [10]. Using second-order Gaussian interpolation, the fading estimate $\hat{y}_{k, i}$ on the data symbol $d_{k, i}$ is obtained by interpolating the fading estimates, $\hat{y}_{k-1,0}, \hat{y}_{k, 0}$ and $\hat{y}_{k+1,0}$, on the pilot symbols, $p_{k-1,0}, p_{k, 0}$ and $p_{k+1,0}$, as follows [4]:

$$
\begin{aligned}
\hat{y}_{k, i}= & C_{-1}(i / L) \hat{y}_{k-1,0}+C_{0}(i / L) \hat{y}_{k, 0} \\
& +C_{1}(i / L) \hat{y}_{k+1,0} \\
C_{-1}(i / L)= & {\left[(i / L)^{2}-i / L\right] / 2 } \\
C_{0}(i / L)= & 1-(i / L)^{2} \\
C_{1}(i / L)= & {\left[(i / L)^{2}+i / L\right] / 2 . }
\end{aligned}
$$

In the study, the SNR is defined as

$$
\mathrm{SNR}=E_{b} / N_{o}=\frac{E_{d} \times(L-1)+E_{p}}{M \times(L-1) \times N_{o}}
$$

where $E_{d}$ and $E_{p}$ are the average transmitted energies per data symbol and pilot symbol, respectively, and $M$ is the number of information bits per data symbol. The signal-to-interference power ratio (SIR) is defined as

$$
\mathrm{SNR}=\sigma_{s}^{2} / \sigma_{c}^{2}
$$


where $\sigma_{s}^{2}$ and $\sigma_{c}^{2}$ are the variances of the Gaussian random variables used to generate the fading processes in the main path and the cochannel, respectively, of Fig. 1, leading to the corresponding channel gains of $2 \sigma_{s}^{2}$ and $2 \sigma_{c}^{2}$. In the tests, the channel gain in the main path has been normalized to unity, i.e., $\sigma_{s}^{2}=$ 0.5 , and hence

$$
\operatorname{SIR}=0.5 / \sigma_{c}^{2}
$$

The main-path to delayed-path power ratio (SDR) is defined as

$$
\mathrm{SDR}=\sigma_{s}^{2} / \sigma_{d}^{2}=0.5 / \sigma_{d}^{2}
$$

where $\sigma_{d}^{2}$ is the common variance of the Gaussian random variables used to generate the delayed-path fading process. The normalized delay between the received signals from the main path and the delayed path is defined as $\tau / T$, where $\tau$ is the delay between the two paths and $T$ is the channel symbol period.

\section{B. BER Performance Versus SNR}

The simulation results on the BER performance of the PSA-16 QAM system using the proposed technique and Gaussian interpolation in the flat fading environments and in the absence of CCI (i.e., SDR $=\infty$ and SIR $=\infty$ ), and with different frame lengths $L$, are shown in Fig. 4. The ideal performance of coherent 16 QAM in the Rayleigh-fading channel is also shown for reference. With a normalized fading rate of $f_{D} T=2 \times 10^{-2}$, Fig. 4(a) shows that the system using Gaussian interpolation with $L=10$ and 15 has high error floors and cannot achieve the practical BER of $10^{-3}$, which is the minimum BER required for voice services of the Universal Mobile Telecommunication System [16]. However, the system using the proposed technique has no error floor under all conditions tested. Comparing the results at the BER of $10^{-3}$, using the proposed technique with $L=10$ and 15 requires 2 and $4.5 \mathrm{~dB}$, respectively, more power than that required for using Gaussian interpolation with $L=5$. However, it should be noticed that with $L=10$ and 15 , the bandwidth redundancies of the system are $10 \%$ and $6.67 \%$, respectively, which are only one-half and one-third of the $20 \%$ redundancy required for $L=5$. Comparing the results with $L=5$, the performance of the proposed technique is about $1 \mathrm{~dB}$ worse than that of Gaussian interpolation at low SNRs; but as the SNR increases, the performance of the proposed technique becomes approaching that of Gaussian interpolation, and eventually becomes better than that of Gaussian interpolation at SNR $>38 \mathrm{~dB}$. With a slower normalized fading rate of $f_{D} T=5 \times 10^{-3}$, Fig. 4(b) shows that even with the Nyquist value of $f_{D} T_{F}=0.5(L=100)$, the system using the proposed technique has no error floor under the SNRs tested. Compared to that of using Gaussian interpolation with $L=10$, using the proposed technique with $L=100$ requires $5 \mathrm{~dB}$ more power to achieve the BER of $10^{-3}$. However, the bandwidth redundancy of the system with $L=100$ is only $1 \%$, which is a decade lower than the $10 \%$ redundancy required for $L=10$.

It can be seen in Fig. 4 that the proposed technique performs significantly better than Gaussian interpolation with longer frame lengths and faster fading rates. This is because the accuracy of the fading estimates obtained using Gaussian
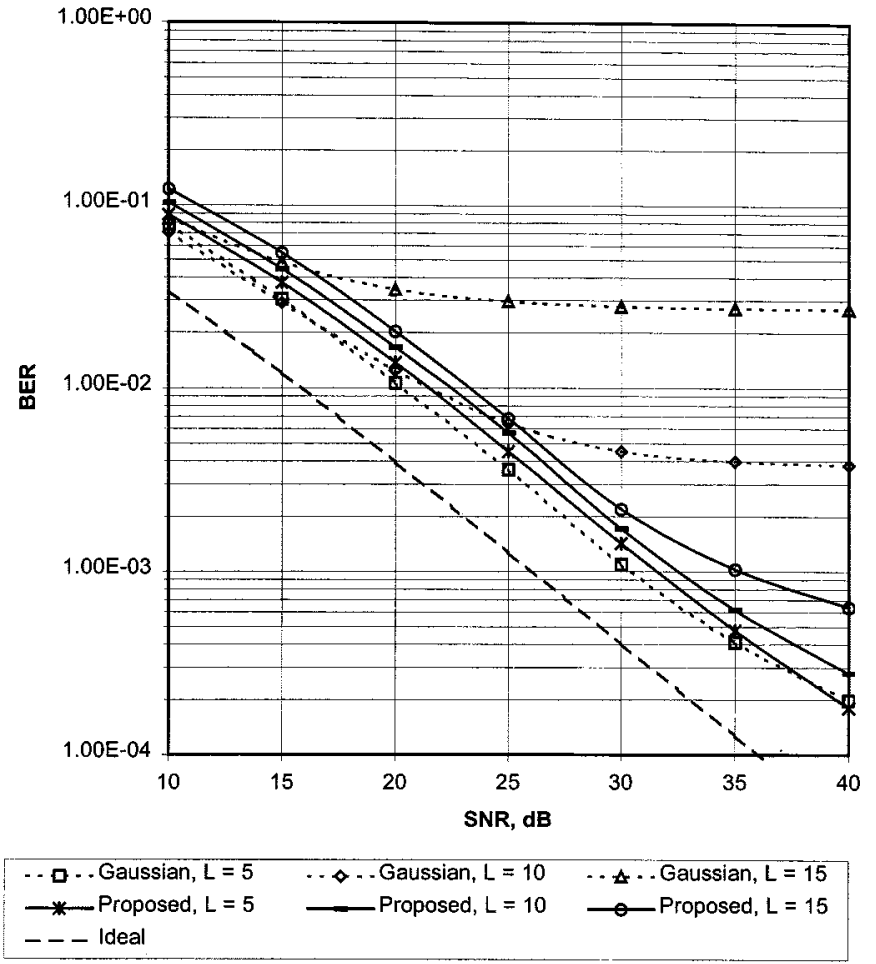

(a)
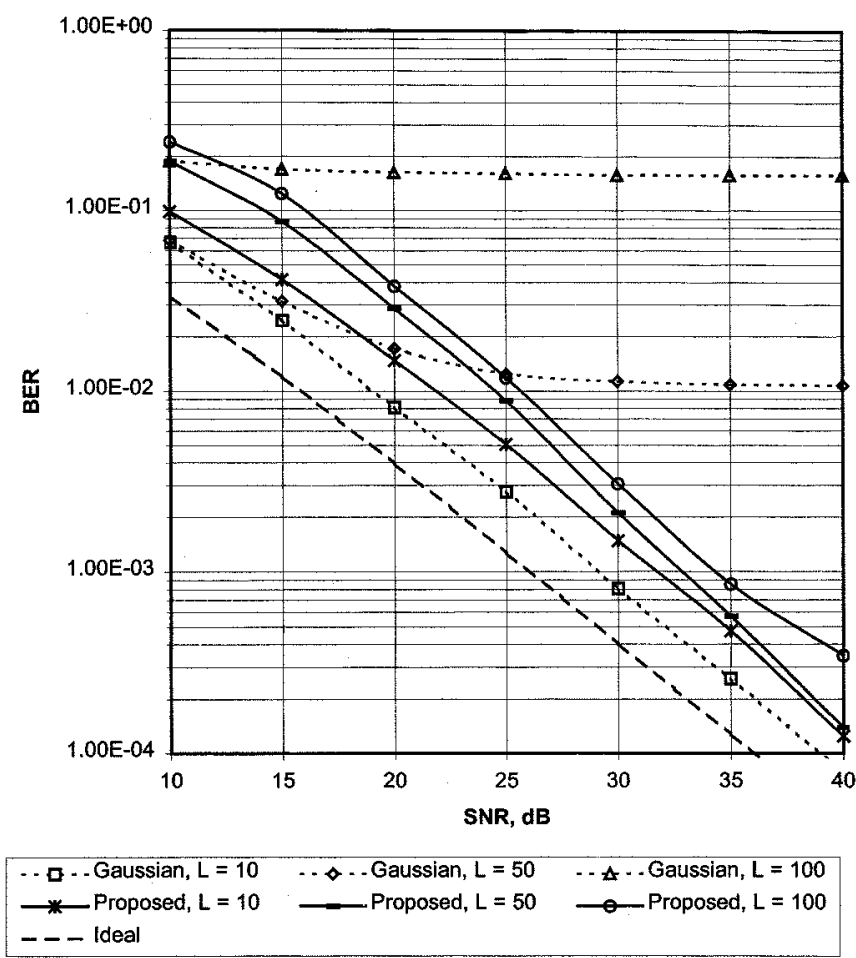

(b)

Fig. 4. BER performance versus SNR, with $\mathrm{SIR}=\infty \mathrm{dB}$ and $\mathrm{SDR}=\infty \mathrm{dB}$, in flat fading channels with (a) $f_{D} T=2 \times 10^{-2}$ and (b) $f_{D} T=5 \times 10^{-3}$.

interpolation on the pilot symbols is degraded by the weakened time correlation between the fading effects on the data symbols and those on the pilot symbols that are used to estimate them. The longer the frame length or the faster the fading rate, the weaker the time correlation and so the less accurate the resultant fading estimates. Since the proposed technique 


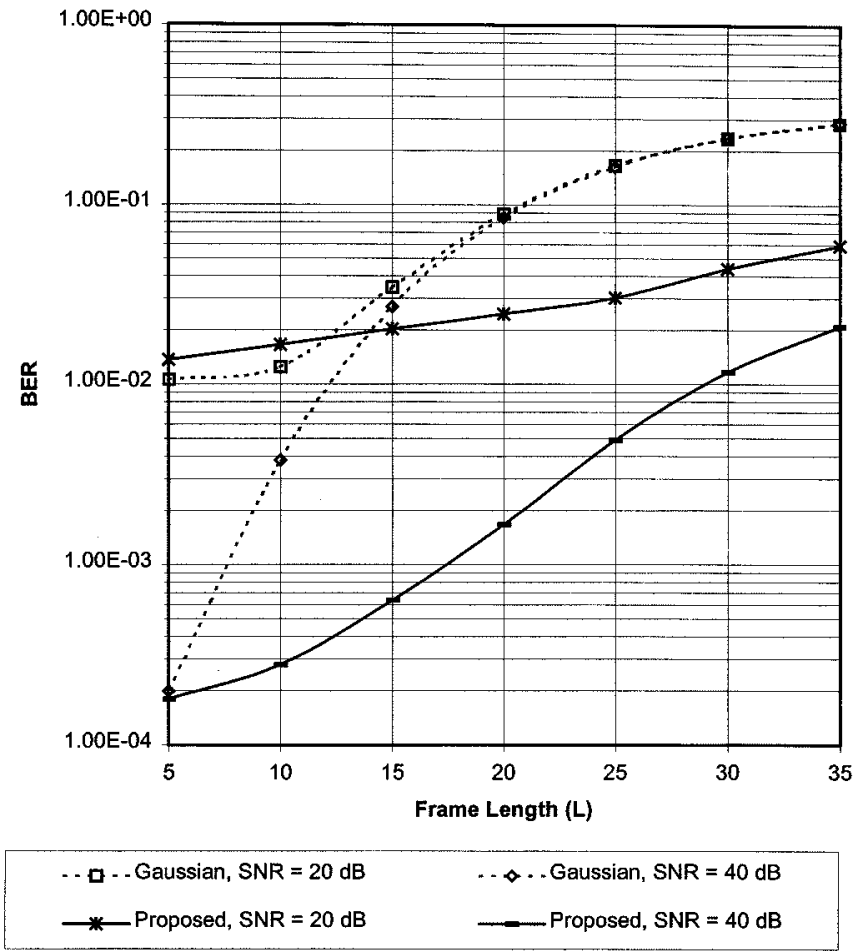

Fig. 5. BER performance versus frame length $L$, with $\operatorname{SIR}=\infty \mathrm{dB}$ and $\mathrm{SDR}=\infty \mathrm{dB}$, in flat fading channel with $f_{D} T=2 \times 10^{-2}$ for SNR $=20$ and $40 \mathrm{~dB}$

employs the detected data symbols as well as the pilot symbols for fading estimation, a stronger time correlation is maintained between the fading effects being estimated and those used to estimate them. Therefore, the resultant fading estimates become more accurate and the system performance can be significantly improved. On the other hand, Fig. 4 shows that the proposed technique is inferior to Gaussian interpolation at low SNRs. This is because, like other data-aided techniques, the performance of the proposed technique is degraded by error propagation resulting from the unreliable data detection at low SNRs. Moreover, since the average transmitted energy per data symbol is less than that per pilot symbol, the estimation error due to additive noise on the data symbols is larger than that of the pilot symbols; therefore, the fading estimates obtained using the data symbols are relatively less accurate and the system performance is degraded. Researchers have suggested using smoothing filters to reduce the noise effects on the fading estimates obtained using the data symbols [11]-[13], but this adds computational load and delay to the system and so is not employed in this study.

To illustrate the effectiveness of the proposed technique in improving the bandwidth efficiency of the system, Fig. 5 shows the BER performance as a function of the frame length $L$, in the flat fading environment with $f_{D} T=2 \times 10^{-2}$ for the SNRs of 20 and $40 \mathrm{~dB}$. It can be seen that as the frame length is increased, the performance of Gaussian interpolation drops off very rapidly, while the performance of the proposed technique has a gradual degradation even when the frame length is increased beyond the Nyquist limit $(L=25)$. Therefore, the proposed technique can be used with much longer frame length

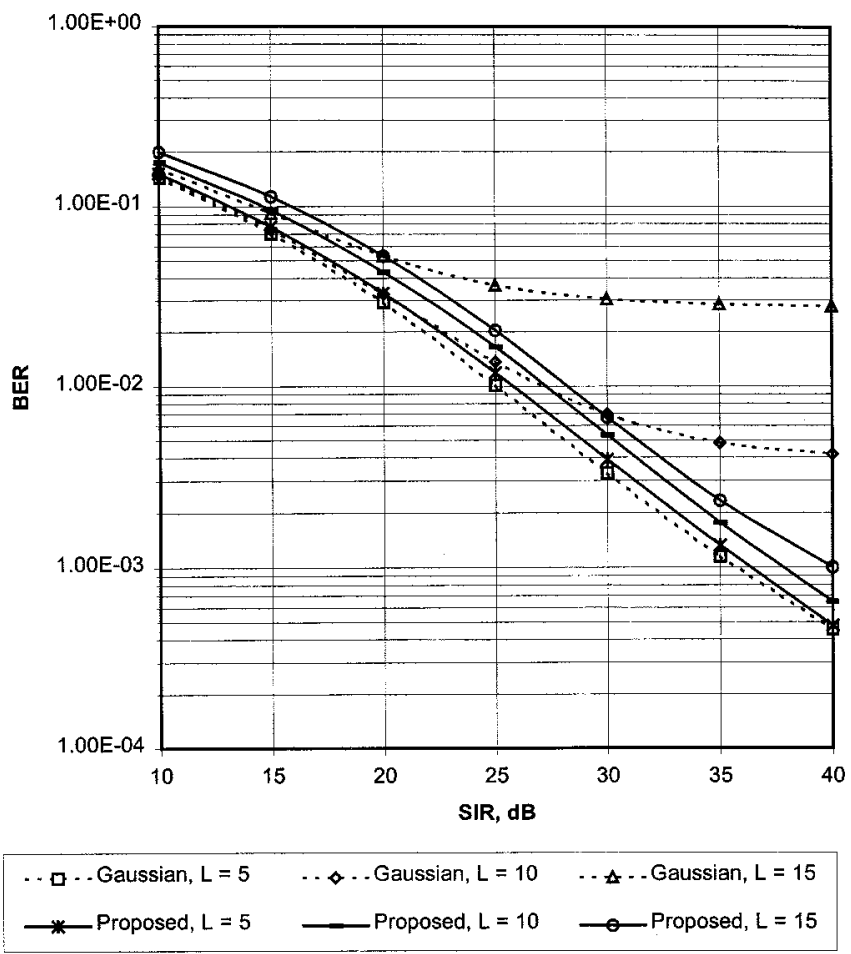

Fig. 6. BER performance versus SIR, with $\mathrm{SNR}=\infty \mathrm{dB}$ and $\mathrm{SDR}=\infty \mathrm{dB}$, in flat fading channel with $f_{D} T=2 \times 10^{-2}$

and still achieve comparable performance as Gaussian interpolation, and thus can greatly improve the bandwidth efficiency of the system. Comparing the results for the SNR of $40 \mathrm{~dB}$ at the BER of $10^{-3}$, the proposed technique can be used with a frame length of $L=17$, while Gaussian interpolation requires a frame length of $L=7$, and thus the bandwidth redundancy can be greatly reduced from $14.3 \%$ to $5.9 \%$.

\section{BER Performance Versus SIR}

To demonstrate the system performance when CCI is the dominant interference as in cellular systems, the BER performance of the PSA-16 QAM system in the flat fading environment with $f_{D} T=2 \times 10^{-2}$ and in absence of AWGN (i.e., SDR $=\infty$ and SNR $=\infty$ ) are shown in Fig. 6. Similar results are obtained compared with those of BER versus SNR, shown in Fig. 4. Comparing the results in Fig. 6 at the BER of $10^{-3}$ indicates that the proposed technique can reduce the bandwidth redundancy of the system from $20 \%(L=5)$ to $10 \%(L=10)$ and $6.67 \%(L=15)$ at the expense of 2 and 4.5 $\mathrm{dB}$, respectively, loss of power efficiency. It can also be seen in Fig. 6 that no error floor occurs using the proposed technique under all conditions tested.

\section{BER Performance Versus SDR}

Finally, the BER performance of the system in the frequencyselective fading environments with $f_{D} T=2 \times 10^{-2}$ and in the absence of CCI and AWGN (i.e., SIR $=\infty$ and SNR $=\infty$ ), with the normalized delay of $\tau / T=0.5$ and $\tau / T=0.25$, are shown in Fig. 7(a) and (b), respectively. It can be seen in both Fig. 7(a) and (b) that the proposed technique can significantly 


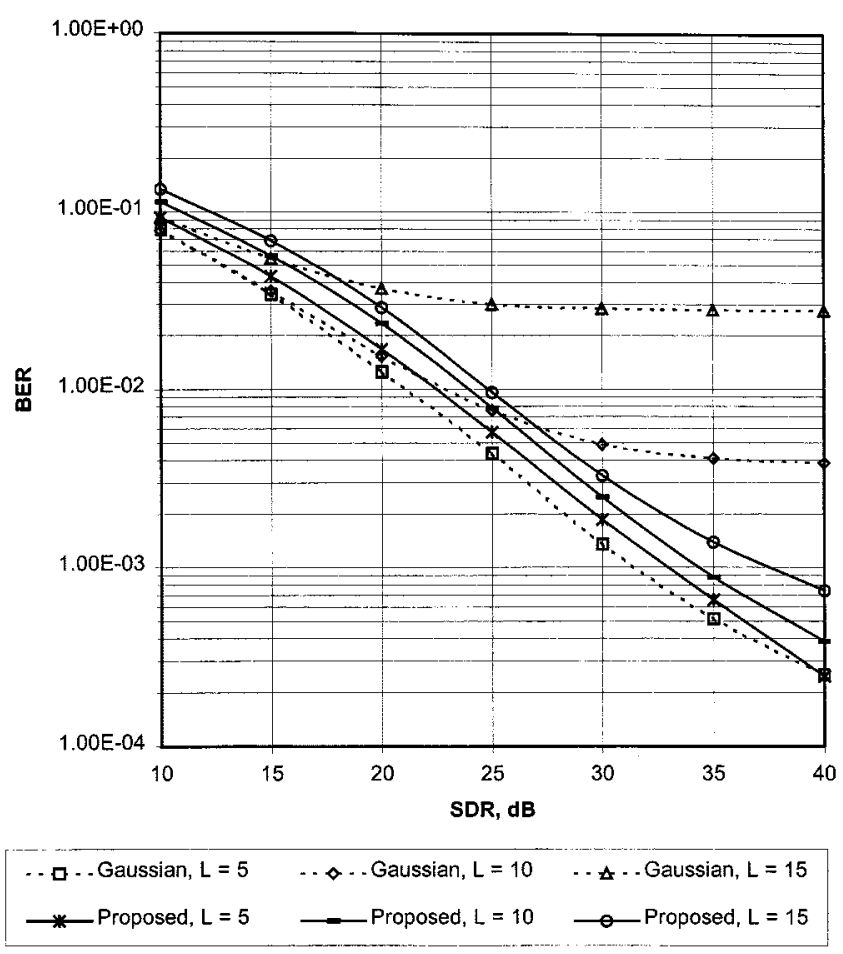

(a)
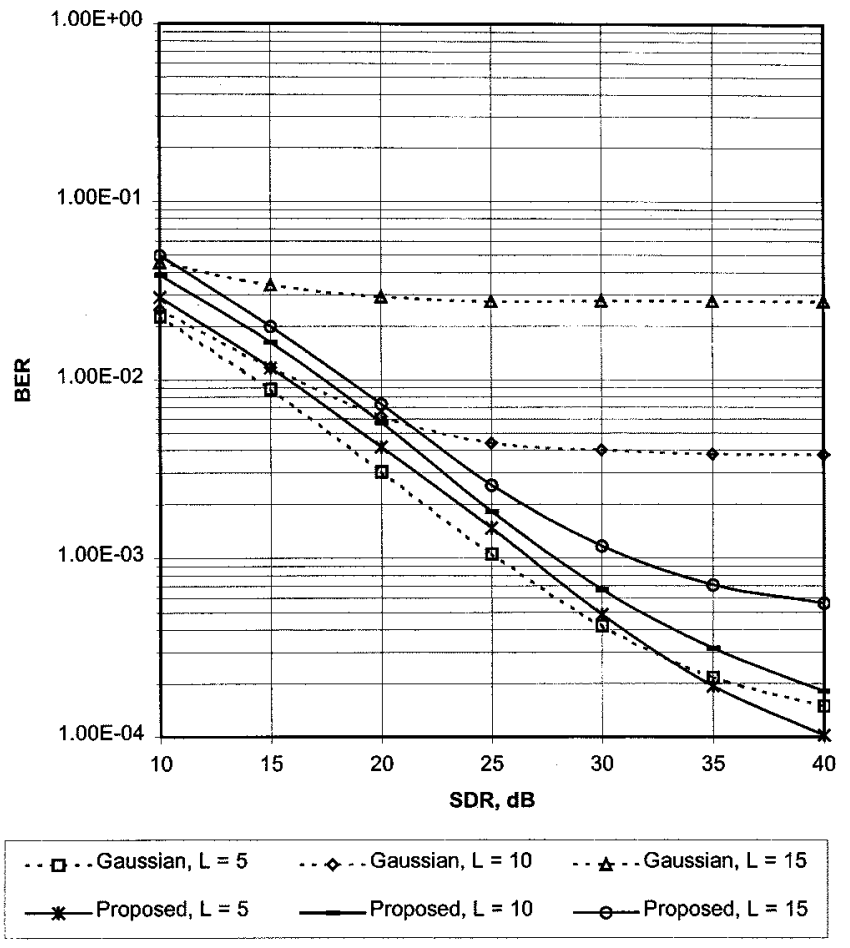

(b)

Fig. 7. BER performance versus SDR, with $\mathrm{SNR}=\infty \mathrm{dB}$ and $\mathrm{SIR}=\infty$ $\mathrm{dB}$, in frequency-selective fading channels with $f_{D} T=2 \times 10^{-2}$, and (a) $\tau / T=0.5$ and (b) $\tau / T=0.25$.

lower the error floors of the system and greatly reduce the bandwidth redundancy from $20 \%$ to $6.67-10 \%$ with 3-6 dB loss of power efficiency.

\section{CONCLUSION}

A bandwidth-efficient fading estimation technique for use in the PSA systems has been proposed and studied by Monte Carlo computer simulation. The proposed technique employs both data and pilot symbols in the estimation process. Since the data symbols are also employed in the estimation process, the pilot symbol rate is no longer constrained by the Nyquist rate and so the bandwidth redundancy of the system can be significantly reduced. Moreover, simple linear interpolation is used to obtain the fading estimates and thus the complexity and latency of the system can be greatly reduced. Results of computer simulation have shown that in both the flat and the frequency-selective Rayleigh-fading environments, the proposed technique can significantly improve the bandwidth efficiency of the PSA system at the expense of a little loss of power efficiency. In the fading environment with $f_{D} T=2 \times 10^{-2}$, the bandwidth redundancy of the system can be reduced from $20 \%$ to as low as $6.67 \%$ with $4.5 \mathrm{~dB}$ loss of power efficiency. It has also been shown that the proposed technique can substantially lower the error floors caused by the use of low-order interpolating functions in the estimation process.

\section{REFERENCES}

[1] M. L. Moher and J. H. Lodge, "TCMP-A modulation and coding strategy for Rician fading channels," IEEE J. Select. Areas Commun., vol. 7, pp. 1347-1355, Dec. 1989.

[2] J. K. Cavers, "An analysis of pilot symbol assisted modulation for Rayleigh fading channels," IEEE Trans. Veh. Technol., vol. 40, pp. 686-693, Nov. 1991.

[3] A. Aghamohammadi, H. Meyr, and G. Ascheid, "A new method for phase synchronization and automatic gain control of linearly modulated signals on frequency-flat fading channels," IEEE Trans. Commun., vol. 39, pp. 25-29, Jan. 1991

[4] S. Sampei and T. Sunaga, "Rayleigh fading compensation for QAM in land mobile radio communications," IEEE Trans. Veh. Technol., vol. 42, no. 2, pp. 137-147, May 1993.

[5] J. K. Cavers and J. Varaldi, "Cochannel interference and pilot symbol assisted modulation," IEEE Trans. Veh. Technol., vol. 42, pp. 407-413, Nov. 1993.

[6] C. L. Liu and K. Feher, "Pilot-symbol aided coherent $M$-ary PSK in frequency-selective fast Rayleigh fading channels," IEEE Trans. Commun., vol. 42, pp. 54-62, Jan. 1994.

[7] J. K. Cavers, "Pilot symbol assisted modulation and differential detection in fading and delay spread," IEEE Trans. Commun., vol. 43, pp. 2206-2212, July 1995.

[8] H. K. Lau and S. W. Cheung, "A pilot symbol-aided technique used for digital signal in multipath environments," Proc. IEEE Int. Conf. Commun., pp. 1126-1130, May 1994.

[9] E. Okamoto, H. B. Li, and T. Ikegami, "Rayleigh fading compensation for QAM by using FFT," in Proc. IEEE PIMRC'96, Taipei, R.O.C., Oct. 1996, pp. 1079-1082.

[10] D. Subasinghe-Dias and K. Feher, "A coded 16 QAM scheme for fast fading mobile radio channels," IEEE Trans. Commun., vol. 43, pp. 1906-1916, May 1995.

[11] G. T. Irvine and P. J. McLane, "Symbol-aided plus decision-directed reception for PSK/TCM modulation on shadowed mobile satellite fading channels," IEEE J. Select. Areas Commun., vol. 10, pp. 1289-1299, Oct. 1992.

[12] Y. Liu and S. D. Blostein, "Identification of frequency nonselective fading channels using decision feedback and adaptive linear prediction," IEEE Trans. Commun., vol. 43, pp. 1484-1492, Feb./Mar./Apr. 1995.

[13] J. P. Seymour and M. P. Fitz, "Two-stage carrier synchronization techniques for nonselective fading," IEEE Trans. Veh. Technol., vol. 44, pp. 103-110, Feb. 1995.

[14] W. C. Jakes, Ed., Microwave Mobile Communications. New York: Wiley, 1974.

[15] J. G. Proakis, Digital Communications. New York: McGraw-Hill, 1995. 
[16] Z. Zvonar, P. Jung, and K. Kammerlander, Eds., GSM: Evolution Toward 3rd Generation Systems. Boston, MA: Kluwer, 1999.

Man-Hung Ng received the B.Sc. degree in computer studies from City University of Hong Kong in 1991, the M.Sc. degree in communication and radio engineering from King's College London, U.K., in 1996, and the Ph.D. degree in mobile communications from the University of Hong Kong in 2001.

He was a Computer Programmer in Hong Kong from 1991 to 1995. He joined the University of Hong Kong as a Research Assistant in 1997. He is now with Lucent Technologies, U.K., as a Standard Engineer.
Sing-Wai Cheung was born in Hong Kong. He received the B.Sc. degree in electrical and electronic engineering from Middlesex University, U.K., in 1982 and the Ph.D. degree from Loughborough University of Technology, U.K., in 1986.

From 1986 to 1988, he was a Postdoctorate Research Assistant in the Communications Research Group of King's College, London University, U.K. During 1988-1990, he was with the Radio and Satellite Communications Division in British Telecom Research Laboratories (now Adastral Park). He joined the Department of Electrical and Electronic Engineering at the University of Hong Kong in 1990 and is now an Associate Professor. He contributes regularly to courses on mobile and satellite communications systems. His current research interests include modulation, coding, fading compensation, and diversity for mobile and satellite communications systems. 